\title{
Adherencia a controles prenatales en el norte argentino desde la perspectiva de la interfaz social
}

\author{
Adherence to prenatal care in the north of Argentina \\ from a social interface perspective
}

Valeria Laura González-Cowes ${ }^{1}$

Fernando Pablo Landini ${ }^{2}$

${ }^{1}$ Facultad de Psicología, Universidad de Buenos Aires. Viamonte 430. 1053 Buenos Aires Argentina. valeria.gonzalezcowes@ gmail.com

${ }^{2}$ Consejo Nacional de

Ciencia y Tecnología.

Buenos Aires Argentina.

\begin{abstract}
Adherence to prenatal care is fundamental for diminishing maternal mortality. Usually, adherence is considered in terms of assisted women's characteristics or the specificities of the health systems and its professionals. Adherence is addressed here as the emergence of the articulation between the rationales and the life realities of physicians and pregnant women. Aiming to understand the adherence to prenatal care, a multiple case study was conducted in the North of Argentina. A total of 47 interviews of pregnant women or women that had been given birth in the last years and to agents of the health system were conducted. The content of the interviews was coded with the support of Atlas Ti software. The research identified two types or interaction between physicians and patients. The first one is based on physicians' representation of assisted women as being ignorant and disinterested, which leads professionals to insist on their recommendations without taking into account problems faced by women. The second part of conceptions is supported in the representation of women as obedient and responsible, which enables a better dialogue between physicians and patients, albeit without the knowledge of a clear interest of the life context of the women being known.

Key words Adherence to medication, Prenatal care, Physician-patient relations, Maternal mortality
\end{abstract}

Resumen La adherencia a controles prenatales constituye una herramienta fundamental para disminuir la mortalidad materna. Usualmente se piensa la adherencia a partir de las características de las mujeres asistidas o de las particularidades del sistema de salud y sus agentes. Aquí se la aborda como emergente del modo en que se articulan las racionalidades y mundos de vida de profesionales y mujeres asistidas. Se realizó un estudio de casos múltiple buscando a comprender la adherencia a controles prenatales de mujeres rurales. Se realizaron 47 entrevistas en el norte argentino a mujeres embarazadas o que habian sido madres en los últimos años y a agentes del sistema de salud. El contenido de las entrevistas fue codificado con el apoyo del software Atlas Ti. El estudio permitió identificar dos tipos de interacción médico-paciente. El primero se caracteriza por una representación de las pacientes como ignorantes y desinteresadas, lo que lleva al agente $a$ insistir con sus recomendaciones sin atender a las dificultades vividas por las mujeres. El segundo parte de concebir a las mujeres como cumplidoras $y$ responsables, lo que permite un mayor diálogo, aún sin que se observe un interés claro por conocer el contexto de vida de las mujeres atendidas.

Palabras clave Cumplimiento de la medicación, Atención prenatal, Relaciones médico-paciente, Mortalidad materna 


\section{Introducción}

En Latinoamérica la tasa de mortalidad materna disminuyó el 40\% entre 1990 y 2013². No obstante, el 95\% de las muertes maternas que aún se producen en la región son prevenibles ${ }^{2}$. En la literatura especializada están ampliamente difundidas diferentes soluciones sanitarias para prevenir la mortalidad materna ${ }^{3,4}$. En general, éstas promueven al acceso universal a atención pre-gestacional, prenatal, durante el parto y durante las primeras semanas del puerperio por parte de personal especializado. En este contexto, los controles prenatales (CPN) constituyen una intervención preventiva por excelencia y un espacio de interfaz entre la mujer embarazada y el sistema de salud local que merece especial atención.

En los CPN se exploran las condiciones de salud de la madre y el niño, se identifican factores de riesgo biológicos y psicosociales, y se brinda orientación a la embarazada sobre el cuidado de su salud y la de su hijo, además de fomentarse hábitos y conductas saludables ${ }^{3,5}$. La OPS ${ }^{2}$ recomienda que en los embarazos de bajo riesgo se realicen al menos cuatro controles periódicos y que éstos comiencen durante el primer trimestre. Sin embargo, el mismo organismo estima que sólo el $46 \%$ de las mujeres rurales embarazadas asisten a un mínimo de cuatro controles en comparación con el $74 \%$ de las mujeres urbanas, observándose también similares niveles de inequidad en mujeres de comunidades pobres, indígenas y afrodescendientes ${ }^{2}$.

A nivel más general diferentes investigaciones llaman la atención sobre las desventajas de las mujeres rurales embarazadas en lo que se refiere a atención prenatal ${ }^{6} \mathrm{y}$ al mayor riesgo de mortalidad materna ${ }^{7}$. Pese a esto, en Latinoamérica son pocos los estudios que abordan la mortalidad materna en zonas rurales en comparación con lo que ocurre en África o el sudeste asiático, donde la mortalidad materna en zonas rurales es una ámbito de estudio por derecho propio ${ }^{8,9}$.

En los estudios sobre CPN, uno de los temas de mayor interés es la falta de adherencia de las embarazadas a las recomendaciones de los profesionales, incluyendo la asistencia a controles con una menor periodicidad a la requerida ${ }^{5,10}$. La bibliografía especializada tiende a abordar esta problemática tanto focalizando en las características de las mujeres como estudiando los factores propios de sistema de salud y/o de sus profesionales asociados a una menor adherencia a controles prenatales (ACPN). Las investigaciones sobre la relación entre las características de las embarazadas y la ACPN suelen poner el foco en (a) variables socio-demográficas como edad, nivel educativo, ocupación, estado civil o religión ${ }^{11}$; (b) la descripción de las creencias, prácticas y hábitos de salud que tienen las embarazadas ${ }^{10}$; (c) las redes de apoyo social con las que éstas cuentan $^{12} ;$ y (d) sus conocimientos sobre CPN y funcionamiento del sistema de salud, así como la importancia que le confieren a ellos ${ }^{13}$.

Los estudios sobre ACPN que abordan aspectos del sistema de salud y de sus agentes tienden a focalizar en dos cuestiones. Primero, en la disponibilidad geográfica de servicios de salud y en las barreras burocráticas o institucionales enfrentadas por las mujeres para obtener atención profesional ${ }^{6,14}$. Segundo, en algunos aspectos de la relación médico-paciente como el trato recibido durante la consulta o la calidad percibida de la atención ${ }^{15,16}$. No obstante, llama la atención que el abordaje del vínculo médico-paciente tienda a incorporar la visión de los profesionales o de las mujeres de manera independiente, sin estudiar la dinámica de la relación en sí misma.

En este contexto, cabe destacar que esta tendencia dicotómica que pone el acento en las características de las embarazadas o en factores asociados al sistema de salud o a sus agentes, tiende a dejar de lado el estudio de los procesos interpersonales que se ponen en juego en la relación médico-paciente en el marco de los CPN. Para abordar estos procesos la noción de interfaz social constituye una herramienta conceptual privilegiada ${ }^{17}$. Según Long ${ }^{18}$ una interfaz social refiere a un espacio en el que interactúan actores sociales (en este caso agentes del sistema de salud y embarazadas) que poseen diferentes experiencias, valores, conocimientos y prioridades, lo que hace probable la existencia de conflictos, así como el establecimiento de relaciones de diálogo, poder y negociación. En esta línea, los CPN pueden ser comprendidos como arenas donde se ponen en juego y "se miden" los mundos de sentido del propio sistema de salud, sus agentes y las mujeres que resultan beneficiarias de las acciones de aquellos. Así, en este artículo se propone abordar la idea de ACPN en tanto resultante o emergente de los mecanismos de negociación y reconfiguración, muchas veces conflictivos, que se producen en la interfaz social que constituyen los CPN. Desde este punto de vista, la ACPN ya no funcionaría como un indicador de la eficacia de la atención médica ${ }^{5}$ o del impacto de factores individuales de las embarazadas asistidas ${ }^{11}$, sino que constituiría un emergente del modo en que se articularon las racionalidades y mundos 
de vida de los actores participantes de la interfaz social que constituyen los CPN. Así, desde esta perspectiva, abordar en profundidad la ACPN obliga a identificar y comprender las dinámicas de interacción entre embarazadas y agentes del sistema de salud que se dan en el contexto de los CPN a partir de las diferentes estrategias, racionalidades y mundos de vida con las que cada uno llega al espacio de interacción, así como el impacto de estas dinámicas en la resolución de problemas específicos relacionados con la salud materna.

En concreto, este trabajo buscará comprender la ACPN desde una perspectiva de interfaz social a partir de un estudio de caso realizado en tres provincias del norte argentino. En este proceso también se apuntará a discutir y contribuir al modo en que es pensada actualmente la ACPN en la literatura internacional.

\section{Metodología}

Se llevó adelante un estudio cualitativo centrado en el análisis de tres estudios de caso realizados en diferentes localidades rurales del norte argentino. En total se realizaron 47 entrevistas semi-estructuradas a pobladoras rurales y a agentes del sistema de salud que trabajan en el ámbito de la salud materna. Las entrevistas se realizaron en las cabeceras municipales (sólo en el caso de agentes del sistema de salud) y en parajes rurales de los municipios de Dos Arroyos (provincia de $\mathrm{Mi}$ siones), Goya (provincia de Corrientes), y de la comuna de Ranchillos (provincia de Tucumán). Las entrevistas, todas grabadas, se distribuyeron del siguiente modo. Dos Arroyos: diez mujeres rurales y siete agentes del sistema de salud. Goya: diez mujeres rurales y cinco agentes del sistema de salud. Ranchillos: nueve mujeres rurales y seis agentes del sistema de salud.

Para ser incluidas en la muestra las mujeres debían residir en los territorios seleccionados, estar embarazadas o ser madres de niños menores de cinco años, haber residido los últimos cinco años en el ámbito rural y pertenecer a una población de bajos ingresos. Por su parte, los agentes del sistema de salud debían trabajar en el sistema público en los territorios seleccionados, poseer título académico vinculado con la tarea que realizan o formación especializada provista por el sistema de salud relacionada con su actividad, atender a población rural en torno a cuestiones vinculadas con salud materna y tener al menos seis meses de experiencia. Las muestras fueron intencionales. Antes de cada entrevista se solicitó un consentimiento informado verbal. En los resultados se identifica a los entrevistados con el siguiente código: 'A' refiere a 'Agentes del sistema de salud', 'M' a 'Mujeres,' 'G' a 'Goya', 'D' a 'Dos Arroyos' y 'R' a 'Ranchillos'. Así, por ejemplo, AR significa 'Agente del Sistema de Salud de Ranchillos'.

Las entrevistas fueron transcriptas y analizadas siguiendo las recomendaciones de Taylor y Bogdan ${ }^{19}$. Primero, se leyeron reiteradamente las transcripciones a fin de identificar temas emergentes asociados a los objetivos propuestos. Luego, con el apoyo del software Atlas Ti se elaboraron categorías para codificar el material en función de los temas identificados, teniendo como referencia los objetivos de la investigación. En la presentación de los resultados dichas categorías de análisis estructuran los diferentes subtítulos.

\section{Resultados}

\section{Contextualización geográfica y organización de los sistemas de salud locales}

En el municipio de Goya las entrevistas se realizaron en los parajes Santa Rita y San Juan. La cabecera municipal cuenta con varios Centros de Atención Primaria de la Salud (CAPS), dos Centros Integradores Comunitarios (CIC) y un Hospital Regional de segundo nivel. Santa Rita se encuentra a $73 \mathrm{~km}$ de la ciudad de Goya, 7 por camino de tierra y el resto por asfalto, mientras que San Juan se ubica a $60 \mathrm{~km}$ por ruta asfaltada. Para todas las zonas rurales del municipio hay cuatro Salas de Atención Primaria de la Salud (SAPS) distribuidas en diferentes parajes. Ellas cuentan con un agente sanitario y son visitadas por médicos cada 7 o 14 días dependiendo del caso. En los parajes sin SAPS las visitas médicas son más espaciadas. En este contexto, las embarazadas pueden recibir un primer chequeo clínico en sus parajes pero luego son derivadas a la ciudad de Goya.

En Misiones las entrevistas se realizaron en el paraje Cerro López, municipio de Dos Arroyos, departamento de Alem. La cabecera departamental dista $25 \mathrm{~km}$ del paraje (entre 1 y $10 \mathrm{~km}$ por camino de tierra, más $20 \mathrm{~km}$ por ruta asfaltada). En ella hay un hospital público de segundo nivel y dos instituciones privadas que realizan partos. El transporte público circula sólo por rutas. En Cerro López hay una posta con un agente sani- 
tario algunos días pero sin visita de médicos. En sus cercanías existen tres CAPS cuya atención se orienta a salud materno-infantil. Éstos prestan servicios médicos uno, dos o tres días por semana según el caso. Allí las pobladoras rurales pueden hacer CPN pero no estudios complementarios. Para el parto viajan a Alem.

En Tucumán el estudio se realizó en el área rural de Ranchillos, cuyo centro urbano se encuentra a $24 \mathrm{~km}$ de la capital provincial. Ranchillos cuenta con un hospital de segundo nivel, ocho CAPS en zonas rurales y un CIC. La distancia máxima de los pobladores rurales a los CAPS es de $6 \mathrm{~km}$, y al Hospital de Ranchillos de entre 6 y $22 \mathrm{~km}$. Sobre la ruta hay transporte público cada 30 minutos, mientras que en los caminos vecinales más alejados éste es más espaciado. En la mayoría de los CAPS hay médicos de distintas especialidades de lunes a viernes, agentes sanitarios y enfermeros que realizan CPN y de puerperio. Para ciertos estudios complementarios las pobladoras acuden Ranchillos, mientras que para otros y para el parto se dirigen a la capital provincial.

\section{Controles prenatales, recomendaciones médicas y respuestas de las pobladoras}

Según las entrevistas, las recomendaciones que los agentes del sistema de salud hacen a las mujeres en los CPN se clasifican en tres grupos. El primero incluye recomendaciones asociadas al protocolo de evaluación y seguimiento del embarazo normal. Éstas involucran la asistencia a controles clínicos y la realización de estudios complementarios como análisis de sangre y orina, ecografías y Papanicolaou (PAP). Estos estudios son descriptos por los agente del sistema de salud como algo que "es obligatorio hacérselo" (AR), lo que resulta consistente con la literatura especializada que destaca la importancia del seguimiento del embarazo y de los estudios prenatales para prevenir muertes maternas ${ }^{20-22}$. Un segundo grupo se relaciona con el uso de medicamentos durante el embarazo. Aquí se engloban la prescripción de fármacos para tratar alguna patología o de suplementos vitamínicos, y la recomendación de evitar el uso de medicamentos sin supervisión médica o de remedios caseros: "desde el momento que vienen le digo 'mamá, no tenés que tomar ninguna medicación sin consultar”' (AR). Por último, el tercer grupo de recomendaciones se dirigen a desalentar ciertas prácticas que las mujeres realizan en su cotidianeidad, como andar en moto o bicicleta, lavar ropa a mano o reali- zar tareas pesadas en el hogar. A esto se suma la recomendación de disminuir el consumo de sal cuando hay retención de líquidos o hipertensión, de evitar fumar y de hacer reposo cuando se requiera.

En relación a las recomendaciones de asistencia periódica a CPN y de realización de estudios complementarios se identifican dos factores que reducen el cumplimiento. Por un lado, aparecen barreras geográficas, económicas y vinculadas al funcionamiento administrativo del sistema de salud, en línea con otros estudios sobre las dificultades de acceso de poblaciones a atención médica $^{13-23}$. Por ejemplo, una mujer destaca dificultades asociadas a tener que viajar para hacerse estudios: "salís hasta la ruta, tenés que andar con la panza y los caminos no te ayudaban nada" (MD). Otra mujer explica que no asistía a controles porque "no tenía plata" para el pasaje (MG). A estas dificultades, muchas veces se suman otras, relacionadas con responsabilidades familiares como no tener con quien dejar a los hijos, lo que no resulta extraño para mujeres rurales que suelen tener muchos niños.

A la vez, también funcionan como obstáculos o facilitadores para la ACPN la evaluación que las mujeres hacen de la utilidad, y/o necesidad de tales controles o estudios. Por ejemplo, las ecografías suele tener gran aceptación puesto que permiten "ver cómo está el bebé" (MD); así como los controles clínicos que apuntan a escuchar los latidos niño o medir el crecimiento del vientre. Explica una entrevistada: "sería lindo que la doctora acá tenga para que te sienta los latidos [...]. Para saber si el bebé está bien" (MR). En contraste, los estudios percibidos como orientados a evaluar la salud de la embarazada suelen postergarse, como los análisis clínicos, el PAP y los controles postparto: "para mí no me interesa tanto como estoy yo [...] lo que a mí me interesa es cómo está mi bebé” (MG).

Las respuestas de las mujeres ante recomendaciones sobre el uso de medicamentos también están atravesadas por ese interés superior en la salud del bebé. Así, es prácticamente unánime evitar el uso de medicamentos no prescriptos, muchas veces justificada por el temor a "que le haga mal al bebé" (MR). Incluso se observa cierta reticencia a tomar suplementos vitamínicos recetados, ya que se los asocia con algo bueno para la embarazada pero que podría afectar negativamente al niño: "por miedo que los pierda a ellos [el embarazo] no quería tomar. Algunas veces los medicamentos que me daba el doctor no quería tomarlos. Esas pastillas para la anemia que toma- 
ba un día, otro día no las tomaba, hasta que las he dejado de tomar" (MR).

Una excepción a este cumplimiento es la utilización de infusiones de hierbas medicinales o los baños de asiento, que se realizan cuando ciertos dolores o malestares no seden luego de ir al médico o cuando se hace imposible acercarse al centro de salud. Otra razón por la que las entrevistadas pueden no seguir recomendaciones relacionadas con la utilización de medicamentos prescriptos es la falta de comprensión de las indicaciones para la toma, ya sea porque no entendieron cuando el médico les explicó, porque no saben leer indicaciones escritas o porque no entienden la letra: "yo no sé leer ni escribir [...] Y a veces a mí me explican y [...] me olvido" (MR).

Por último, las recomendaciones que apuntan a desalentar ciertas prácticas de las mujeres durante el embarazo son las más afectadas por las situaciones de vida de cada entrevistada. Se observa que el cumplimiento de estas indicaciones baja notablemente cuando requieren que las mujeres dejen tareas que sienten como su responsabilidad (como atender a sus hijos), que asumen como impostergables, o cuando perciben que no tienen otros medios para subsanar el abandono de una práctica (no pueden acercarse al CAPS si no van en moto, o no hay quien acarree el agua en lugar de ellas). Una de las entrevistadas lo explica: "[el médico] me decía que yo no tenía que hacer fuerza, pero yo no podía hacer reposo. ¿Cómo iba a hacer? Tenía que acarrear agua sí o sí, lavar la ropa" (MD).

En este contexto también cabe mencionar que muchas mujeres no comentan al médico estas dificultades: "no, yo no le decía [de los problemas para seguir las recomendaciones] porque si uno dice... da lo mismo que no decir" [MD]. Al mismo tiempo, se observa que los profesionales se interesan poco por las posibilidades reales de cumplimiento de estas mujeres, presentando las recomendaciones como la única opción posible.

\section{Comprensiones mutuas y dinámicas de interacción médico-paciente}

La perspectiva de los agentes del sistema de salud. Los agentes del sistema de salud tienden a comprender a las mujeres rurales y a sus conductas a partir de tres tipos diferenciados de representaciones. El primer núcleo, el más frecuente, describe a las embarazadas a partir de rasgos internos negativos e inmodificables que explicarían, a los ojos de estos profesionales, su baja ACPN. En este sentido, se las suele describir como irresponsables, pasivas o poco interesadas en su salud y en su embarazo. Un profesional señala: "no sé qué es lo que hacen, ni siquiera en los controles nos han llegado, para ellas está todo bien. Nosotros les decimos, 'te tenés que ir a controlar'. Y no, nada. Así es la mentalidad” (AG). También se tiende a describir a las mujeres como ignorantes o con dificultades para comprender: "depende de cada paraje. En algunos son muy ignorantes que no entienden lo que le decís por más que le expliques" (AG). Al mismo tiempo, también de habla de ellas como tímidas, retraídas o con falta de fluidez para la palabra: "no son de preguntar [...] porque generalmente acá no tenemos la escuela primaria, incompleta, la mayoría de las mujeres, las que vienen" (AD).

Así, se configura una imagen cristalizada de las mujeres asistidas que se carga de contenidos internos de carácter negativo, la cual es utilizada para explicar sus conductas. En el fondo, lo que subyace es que ellas 'son así por su naturaleza', lo que dificulta la posibilidad de comprender las circunstancias difíciles que viven estas mujeres, como por ejemplo el no encontrar con quién dejar a los hijos, la falta de transporte o la carencia de medios económicos para llegar a los servicios sanitarios. De esta manera, prevalece una explicación basada en carencias inherentes en lugar de abrirse a la comprensión de la complejidad del contexto.

Así, se observa que los agentes del sistema de salud se comprenden a sí mismos como expertos $\mathrm{y}$ a las pacientes como personas pasivas, ignorantes o con falta de interés, lo que delinea una estrategia de interacción en la cual los primeros buscan estereotipadamente que las mujeres hagan o entiendan lo que sostiene la perspectiva médica. Un profesional lo ejemplifica: "hasta que no tenga la certeza de que entendió no le dejo ir, [...] le explicás, le explicas y le explicas" (AD). De esta forma, se construye una dinámica circular en la cual los agentes del sistema de salud insisten en su punto de vista sin atender al contexto y a las circunstancias de vida de las mujeres. Así, éstas parecen reaccionar con pasividad y desidia, lo que lleva al médico a seguir insistiendo, favoreciéndose sentimientos de frustración: "y no me dan muchas chances como para... entonces uno por ahí termina ya cansándose un poco de explicar la importancia y que del otro lado haya apatía” (AR).

El segundo núcleo de representaciones usado por los profesionales para comprender las acciones de las mujeres también explica las dificultades de ACPN a partir de características persona- 
les internas negativas. No obstante, en este caso se las considera modificables. Aquí se tiende a hablar de la falta de comprensión de las mujeres, así como de la limitación de sus conocimientos para el cuidado de la salud durante el embarazo: "hay gente que... no van a la escuela, eso mismo hace que no tengan la responsabilidad sobre su salud, entonces eso hace que carezcan de conocimiento" (AG). En este contexto, los agentes del sistema de salud tienden a repetir la estrategia de vinculación descripta anteriormente. No obstante, en este caso existe expectativa y confianza en la posibilidad de cambio, lo que fortalece el interés por desarrollar estrategias didácticas o pedagógicas que puedan resultar de utilidad: "una consulta en una ubicación rural te demanda el doble porque $[. .$.$] le tenés que escribir con mayúscula,$ letra grande, por ahí usar otros parámetros de referencia [en vez de] 'cada 12 horas' decirle 'a la mañana cuando sale el sol tomás'” (AG).

Por último, con muchas menos menciones, aparece un tercer grupo de representaciones que incluye referencias a las pacientes rurales como cumplidoras y colaboradoras que "ya vienen mentalizadas con que tienen que hacerse el control". De esta manera, se abre la posibilidad de una mayor interlocución, donde no haya búsqueda de imponer una práctica o una forma de ser sino de comprender los problemas que se susci$\tan$ en relación a las recomendaciones que hacen los profesionales. No obstante, dado que la mayor parte de las descripciones focalizan en el carácter 'cumplidor' de las mujeres, debe tenerse cuidado con asumir esto como expresión de un vínculo más horizontal o donde exista mayor reconocimiento de la perspectiva y situación de la gente, ya que fácilmente estas características podrían no ser más que una respuesta a la pasividad de estas mujeres frente a profesionales dominantes: "la que te dice 'salgo como pueda' generalmente es más humilde y más ignorante [...] porque es como que lo que vos decís lo acata" (AR).

La perspectiva de las pacientes. En los discursos de las mujeres se observan dos tipos de descripciones de los agentes del sistema de salud. El primer grupo de representaciones describe a los profesionales en términos de buen trato y de otras características positivas. Así, se habla de los médicos como personas que se ocupan de las necesidades de la gente y se interesan por lo que les pasa: "la doctora es muy buena, me atiende bien $[\ldots]$ siempre me atiende bien $[\ldots]$ me pregunta cómo estaba [mi otro hijo]" (MR). A la vez, también se señala que los agentes del sistema de salud procuran resolver las necesidades de sus pacien- tes sin estar específicamente obligados: "a veces, no podés comprar un remedio y te dicen 'ya te voy a conseguir"' (MR). Aquí, resulta interesante mencionar que estas representaciones positivas, muchas veces asociadas a la empatía de los profesionales, van en línea con la importancia dada por la bibliografía médico-paciente a la construcción de un buen vínculo interpersonal ${ }^{24,25}$.

Si bien cuantitativamente algo menos importante, también existe un segundo grupo de representaciones que se estructura a partir de quejas y reclamos sobre los agentes del sistema de salud. Aquí se observan tres elementos. Primero, referencias a la falta de conocimientos y capacidad de los médicos: "pero el doctor no se animaba. Supuestamente, si es ginecólogo, ha atendido partos [...] Entonces, ¿qué va a hacer él cuando llegue una mujer ya con el hijo saliendo ahí? Se va a poner entre la espada y la pared y no va a saber cómo manejar" (MR). En segundo lugar, también se señala que los profesionales no se hacen entender, no explican bien o no tienen voluntad para hacerlo: "a veces, un médico te habla como si fuese que ellos están estudiando y creen que nosotros vamos a saber lo que ellos te están diciendo" (MR). A la vez también hay mujeres que señalan que los médicos no escuchan a los pacientes o no se interesan por ellos: "ella decía que [el malestar] era del viaje y no me revisó nada. Y después $[\ldots]$ volvimos y ahí ella me revisó y me internó" (MD).

Dinámicas vinculares. Estos dos tipos de representaciones de las mujeres rurales respecto de los agentes del sistema de salud parecen inducir dos tipos diferentes de interacción. Por una parte, las representaciones de los agentes del sistema de salud como personas capaces, con buen trato, que se ocupan de sus pacientes, parecen invitar a las mujeres a buscar y a continuar con la atención médica, en el marco de una relación de confianza: "yo no lo tomo como un doctor sino como otra persona que está conversando conmigo" (MR). Esto parecería articularse con la visión que los profesionales tienen de ellas como personas 'cumplidoras', lo que coloca a las mujeres en una posición pasiva, de espera 'paciente', en la cual el vínculo y las recomendaciones son estructuradas exclusivamente a partir de la perspectiva de los agentes del sistema de salud.

Por su parte, las representaciones negativas sobre los profesionales parecieran estar en la base de dos conductas. Primero, el escaso interés por buscar asistencia médica. No obstante, lo que pareciera primar aquí es buscar asistencia pero desde una posición de 'resistencia pasiva', en la cual se 
asiste a la consulta pero sin un compromiso real con la interacción, dado que no se abren espacios para que las mujeres puedan compartir sus experiencias y problemas prácticos cotidianos, los cuales usualmente no son tenidos en cuenta por los profesionales. Claramente, esta segunda dinámica de interacción se articula con la percepción de desidia y desinterés que los agentes del sistema de salud identifican en las mujeres rurales. En las Figuras 1 y 2 pueden observarse estos dos tipos diferenciados de interacción entre agentes del sistema de salud y mujeres en el contexto de la salud materna.

\section{Discusión}

En este trabajo se abordó la ACPN desde la perspectiva de la interfaz social. En este proceso, se arribó a diferentes resultados de interés. A nivel empírico, el resultado de mayor valor refiere a la identificación de dos dinámicas de interacción diferenciadas entre agentes del sistema de salud y mujeres rurales. A partir de la metodología utilizada, se observó que estas dinámicas de interacción se apoyan en diferentes representaciones mutuas. Esta tipología de dinámicas de interacción, así como la descripción de las representaciones y supuestos en los que éstas se sostienen, constituyen un aporte original a la literatura referida tanto a salud materna como a la relación médico-paciente. En efecto, si bien en diferentes trabajos se hace énfasis en la necesidad de un buen vínculo entre ambos ${ }^{24,25}$, la identificación de tipos específicos de interacción sostenidos en representaciones mutuas no es algo que haya sido identificado en la bibliografía académica disponible.

Resulta importante resaltar que estos diferentes tipos de interacción poseen impactos di-

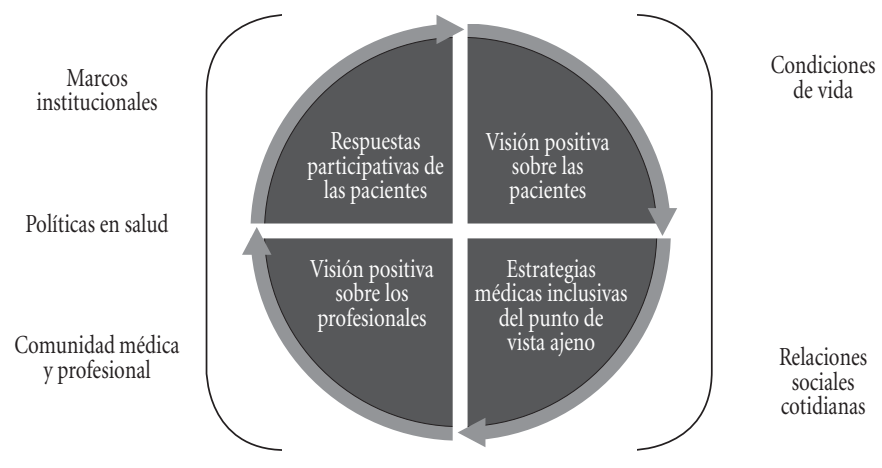

Figura 1. Dinámicas de interacción positivas médico-paciente.

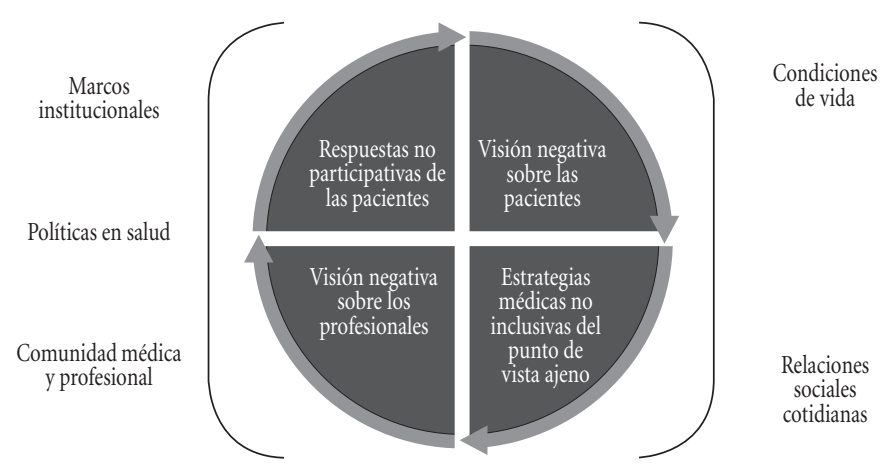

Figura 2. Dinámicas de interacción negativas médico-paciente. 
ferenciales en la ACPN, lo que resulta de interés para la prevención de la mortalidad materna. El primer tipo de interacción se articula en torno a la representación de los agentes del sistema de salud como personas capaces y de buen trato, y de las mujeres como obedientes y responsables. En este caso, si bien se tiende a establecer una relación vertical centrada en la lógica médica, el buen vínculo interpersonal facilita que las mujeres busquen continuar con los controles prenatales. A la vez, la cordialidad de la relación también pareciera permitir que las mujeres planteen problemas para cumplir con lo recomendado. Así, se aumenta la probabilidad de que se construyan recomendaciones superadoras, entendidas como a aquellas que, además de tener en cuenta la situación médica de las pacientes, también consideran el contexto material, cultural y subjetivo en el que deben aplicarse, incrementando su cumplimiento.

Por su parte, el segundo tipo de vínculo identificado se sostiene en representaciones negativas sobre las mujeres y sus capacidades y de los profesionales como personas con conocimientos limitados y con falta de interés por sus pacientes. Esto lleva a una dinámica de interacción en la cual los agentes del sistema de salud tienden a repetir insistentemente las mismas recomendaciones, mientras que las mujeres callan sus problemas prácticos para cumplir con ellas desde una posición de 'resistencia pasiva'. Así, ni los agentes del sistema de salud se abren a comprender las condiciones contextuales que limitan la posibilidad de las mujeres de adherir a los CPN $\mathrm{y}$ a diferentes recomendaciones relacionadas con ellos, ni las propias pacientes comparten sus dificultades con los profesionales al sentir que no tienen lugar para ello. En este contexto, caracterizado por el monólogo, sólo una perspectiva y un único punto de vista tiene lugar: el médico. De esta manera, la ACPN disminuye, ya que las mujeres no se sienten impulsadas a continuar con los controles, a la vez que las recomendaciones de los profesionales no tienen en cuenta variables que limitan su posible cumplimiento.

Analizando ambos tipos de interacción, si bien en el primero aparece una mayor apertura al reconocimiento de la situación contextual y al punto de vista de las mujeres, resulta llamativo que en ninguno de los dos aparezca como aspecto destacado la necesidad de comprender (por parte de los agentes del sistema de salud) qué es lo que lleva a las mujeres a una baja ACPN y a recomendaciones relacionadas. Así, se observa que en ambos casos el vínculo médico-paciente termina estructurándose de manera jerárquica, sin que las beneficiarias, sus problemas y su perspectiva sean consideradas como factor legítimo que debiera entrar en diálogo con el conocimiento profesional para generar 'recomendaciones superadoras'. En consecuencia, también podría decirse que si bien fueron descritos dos tipos de interacción entre agentes del sistema de salud y mujeres beneficiarias, también fue identificado un tercer tipo posible (aunque empíricamente ausente), sostenido en el reconocimiento del punto de vista y la situación concreta de las mujeres, y caracterizado por un diálogo horizontal en el cual el agente del sistema de salud es experto en términos médicos, mientras que las beneficiarias son portadoras de un contexto, una experiencia y un marco cultural que debe ser tenido en cuenta.

Atentos a la ausencia de este tercer tipo de interacción, podría pensarse que esto se debe a que los agentes del sistema de salud carecen de un repertorio ${ }^{18}$ de conductas y de representaciones que les permitan generar este tipo de vínculo, en el que el punto de vista y la situación de vida del paciente es buscado activamente en el contexto de una relación más horizontal. En consecuencia, los agentes del sistema de salud terminan quedando atrapados entre repetir fallidamente recomendaciones que resultan inefectivas (en el sentido de tener baja adherencia) y buscar una relación más fluida pero sin herramientas para que ese mejor vínculo se convierta en una comprensión más profunda de las razones de la limitada ACPN en los contextos en los que trabajan.

Los resultados y reflexiones presentados hasta este momento poseen importantes consecuencias para la formación de los agentes del sistema de salud y la conformación de los equipos que trabajan en salud materna en territorios rurales. Respecto de la formación de los agentes del sistema de salud, se hace evidente la necesidad de reconsiderar la importancia de incluir el desarrollo de capacidades para la identificación de diferencias culturales y para la comprensión de la perspectiva (prioridades, intereses, etc.) y situación material de los pacientes de áreas rurales, así como para lidiar con estas diferencias y situaciones de manera eficaz ${ }^{26,27}$. A nivel de la conformación del equipo de salud, una consecuencia adicional es el reconocimiento del potencial que tienen los promotores y agentes sanitarios para funcionar como mediadores culturales en la interfaz social que constituyen los $\mathrm{CPN}^{28}$. En concreto, ellos pueden ayudar a los médicos a comprender mejor la situación y perspectiva de sus pacientes (con quienes usualmente comparten el entorno 
cotidiano), y a las mujeres a contextualizar las recomendaciones realizadas por los médicos.

A nivel conceptual, este artículo también ha propuesto una forma novedosa de pensar la ACPN a partir del concepto de interfaz social. Usualmente, la literatura académica tiende a pensar la adherencia en términos de las características individuales de las mujeres asistidas o, en su defecto, las particularidades del sistema de salud y de sus profesionales ${ }^{29,30}$. En paralelo, también existen algunos estudios que destacan la incidencia de factores de índole cultural en la $\mathrm{ACPN}^{31}$. No obstante, las dinámicas de interacción entre agentes del sistema de salud y pacientes han recibido escasa atención. En contraste, en este estudio se ha presentado un marco conceptual específico para pensar la ACPN que entiende a la adherencia como emergente del modo en que se resuelven los quiebres existentes entre las pers- pectivas, experiencias y mundos de vida de los actores que forman parte de la interfaz social de atención médica en el contexto de la salud materna. Así, este artículo también ha aportado a nivel conceptual al estudio de las dinámicas de interacción médico-paciente, a la vez que ha echado luz sobre la incidencia de las diferencias en las miradas de ambos en la ACPN, abriendo la puerta al estudio de factores usualmente no considerados.

Por último, cabe señalar que si bien este trabajo ha aportado a la literatura académica existente sobre ACPN, lo ha hecho desde un estudio de casos múltiple, lo que establece límites a su posibilidad de generalización. En este sentido, no se considera que los resultados alcanzados sean generalizables, sino más bien que tienen potencialidad para ayudar a pensar la incidencia, en contextos específicos, de factores usualmente no tenidos en cuenta por los estudios tradicionales de ACPN.

\section{Colaboradores}

VL González-Cowes participó en el relevamiento de las entrevistas y la categorización de todas las transcripciones, tarea que fue supervisada y orientada por FP Landini, quien también aporto activamente a la interpretación y discusión de los datos. El artículo fue redactado de manera conjunta, siendo responsabilidad de FP Landini la revisión crítica de todo el manuscrito. La versión final fue aprobada por ambos autores.

\section{Referencias}

1. Organización Mundial de la Salud (OMS). Trends in maternal mortality estimates 1990 to 2013. Estimates by WHO, UNICEF, UNFPA, The World Bank and the United Nations Population Division. 2014 [acceso 2016 Abr 11]. Disponible en: http://apps.who.int/iris/bitstr eam/10665/112682/2/9789241507226_eng.pdf?ua=1

2. Organización Panamericana de la Salud (OPAS). Plan de acción para acelerar la reducción de la mortalidad materna y la morbilidad materna grave. 2011 [acceso 11 abr 2016]. Disponible en: http://www.paho.org/col/ index.php?option $=$ com_docman\&task $=$ doc_download\&gid=1238\&Itemid=

3. Fescina R, De Mucio B, Díaz-Rosello, J, Martínez G, Seruya S, Durán P. Salud sexual y reproductiva: guías para el continuo de atención de la mujer y el recién nacido focalizada en APS. Montevideo: CLAP/SMR; 2011.

4. Pazmiño de Osorio S, Guzmán-Gómez N. Control prenatal y su impacto en la mortalidad materna: Análisis de una tendencia, 1994-2004. Cali, Colombia. Rev Colomb Obstet Ginecol 2009; 60(1):12-18.

5. Cáceres-Manrique FM. El control prenatal: una reflexión urgente. Rev Colomb Obstet Ginecol 2009; 60(2):165-170.

6. Alexandre PK, Saint-Jean G, Crandall L, Fevrin E. Prenatal care utilization in rural areas and urban areas of Haiti. Rev Panam Salud Publica 2005; 18(2):84-92. 
7. Scheil-Adlung X. Global evidence on inequities in rural health protection: new data on rural deficits in health coverage for 174 countries. Ginebra: OIT; 2015.

8. Adamson P, Krupp K, Niranjankumar B, Freeman A, Khan M, Madhivanan P. Are marginalized women being left behind? A population-based study of institutional deliveries in Karnataka, India. BMC Public Health [Internet] 2012 [acceso $2016 \mathrm{Abr} 11$ ]; 12:30. Disponible en: www.biomedcentral.com/1471-2458/12/30

9. Iyengar K, Iyengar D, Suhalka V, Dashora D. Pregnancy-related deaths in rural Rajasthan, India: Exploring causes, context, and care-seeking through verbal autopsy. J Health Popul Nutr 2009; 27(2):293-302.

10. Valadez I, Alfaro N, Pozos E. Cuidados alternativos en la atención del embarazo en Jalisco, México. Cad Saude Publica 2003; 19(5):1313-1321.

11. Calle L, Pardo R, Solís G, Lombraña E, Pérez C, González MT. Gestación sin controlar y su impacto socio-sanitario en una población pediátrica. Bol Pediatr 2013; 53(223):28-36.

12. Guimarães EC, Melo ECP. Características do apoio social associados à prematuridade em uma população de puérperas de baixa renda. Esc Anna Nery 2011; 15(1):54-61.

13. Figueiredo PP, Rossoni E. O acesso à assistência pré-natal na atenção básica à saúde sob a ótica das gestantes. Rev Gaucha Enferm 2008; 29(2):238-425.

14. Seclen JA, Benavides B, Jacoby E, Velásquez A, Watanabe E. Existe una relación entre los programas de mejora de la calidad y la satisfacción de usuarias de atención prenatal?: experiencia en hospitales del Perú. Rev Panam Salud Publica 2004; 16(3):149-157.

15. Almeida CAL, Tanaka OY. Perspectiva das mulheres na avaliação do Programa de Humanização do Pré-Natal e Nascimento. Cad Saude Publica 2009; 43(1):98-104.

16. Hilgert NI, Gil GE. Reproductive medicine in northwest Argentina: traditional and institutional systems. $J$ Ethnobiol Ethnomed [Internet] 2007 [acceso $2016 \mathrm{Abr}$ 11]; 3:19. Disponible en: http://ethnobiomed.biomedcentral.com/articles/10.1186/1746-4269-3-19

17. Landini F, González-Cowes V, D’Amore E. Hacia un marco conceptual para repensar la accesibilidad cultural. Cad Saude Publica 2014; 30(2):231-244.

18. Long N. Sociología del desarrollo: una perspectiva centrada en el actor. México: CIESAS; 2007.

19. Taylor S, Bogdan R. Introducción a los métodos cualitativos de investigación. La búsqueda de significados. Barcelona: Paidós; 1987.

20. Álvarez JL, Gil R, Hernández V, Gil A. Factors associated with maternal mortality in Sub-Saharan Africa: an ecological study. BMC Public Health [Internet]. 2009 [acceso 2016 Abr 11]; 9:462. Disponible en: http://bmcpublichealth.biomedcentral.com/articles/10.1186/1471-2458-9-462
21. Supratikto G, Wirth ME, Achadi E, Cohen S, Ronsmans C. A district-based audit of the causes and circumstances of maternal deaths in South Kalimantan, Indonesia. Bull World Health Organ 2002; 80(3):228-234.

22. Távara-Orozco L. Manejo de las principales causas de mortalidad materna. Acta Med Peruana 2005; 22(2):6364.

23. Landini F, González-Cowes V, Bianqui V, Logiovine, S, Vázquez J, Viudes S. Accesibilidad en el ámbito de la salud materna de mujeres rurales de tres localidades del norte argentino. Saude Soc 2015; 24(4):1151-1163.

24. Herrera N, Gutiérrez, M, Ballesteros M, Izzedin, R, Gómez A, Sánchez, L. Representaciones sociales de la relación médico paciente en médicos y pacientes en Bogotá, Colombia. Rev Salud Pública (Bogota) 2010; 12(3):343-355.

25. Weissmann P, Haidet P, Branch W, Gracey C, Frankel R. Teaching humanism on the wards: What patients value in outstanding attending physicians. J Commun Healthc 2010; 3(3-4):291-299.

26. Houle C, Harwood E, Watkins A, Baum K. What women want from their physicians: A qualitative analysis. J Womens Health 2007; 16(4):543-550.

27. Shukla A, Yadav V, Kastury N. Doctor-patient communication: An important but often ignored aspect in clinical medicine. J Indian Acad C Med 2010; 11(3):208-211.

28. Gomes K, Cotta R, Mitre S, Batista R, Cherchiglia M. O agente comunitário de saúde e a consolidação do Sistema Único de Saúde: reflexões contemporâneas. Physis 2010; 20(4):1143-1164.

29. Furfaro K, Pécora A, Jankovic MP, Llompart V, Cantero A, San-Martín ME. Control prenatal tardío: ¿barreras en el sistema de salud? R Hosp Mato Inf R Sardá 2008; 27(3):114-119.

30. Cano-Serral G, Rodríguez-Sanz M, Borrell C, Pérez MM, Salvador J. Desigualdades socioeconómicas relacionadas con el cuidado y el control del embarazo. Gac Sanit 2006; 20(1):25-30.

31. Lasso-Toro P. Atención prenatal: ¿tensiones o rutas de posibilidad entre la cultura y el sistema de salud? Pensam Psicol 2012; 10(2):123-133.

Artigo apresentado em 11/05/2016

Aprovado em 05/09/2016

Versão final apresentada em 07/09/2016 